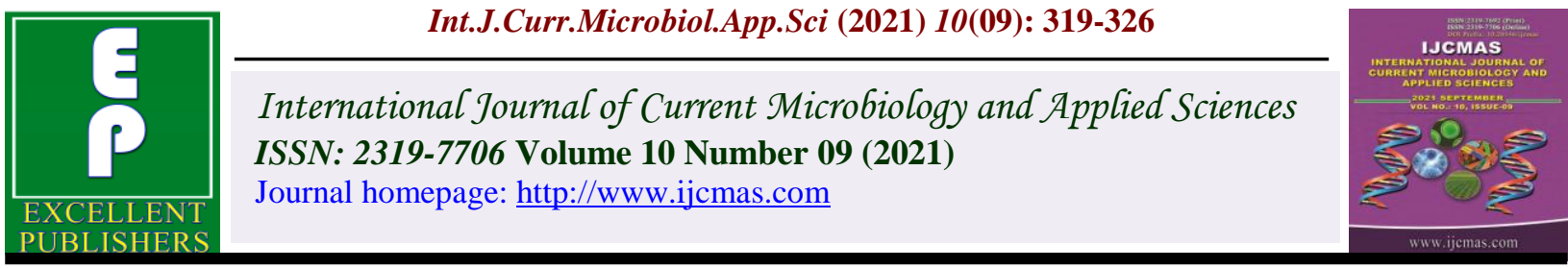

\title{
Influence of Intercropping on Larval Population of Chickpea Pod Borer, Helicoverpa armigera (Hub.) in Chickpea (Cicer arietinum L.)
}

\author{
Hari Prakash Namdev ${ }^{1}$, Ram Subhag Singh ${ }^{1}$ and Bharat Lal Kushwaha ${ }^{2}$ \\ ${ }^{1}$ Department of Agriculture Entomology, ${ }^{2}$ Department of Agronomy, Brahmanand Post \\ Graduate College, Rath, Hamirpur Uttar Pradesh - 210431, India
}

*Corresponding author

\begin{tabular}{|l|}
\hline Ke y w o r d s \\
Chickpea, \\
intercropping, \\
Helicoverpa \\
armigera (Hub.) \\
population
\end{tabular}

A field experiment on influence of intercropping on the larval population of chickpea pod borer, Helicoverpa armigera (Hub.) in chickpea was conducted at Agricultural Research Farm of Brahmanand Post Graduate College, Rath, Hamirpur, Uttar Pradesh during 2016-17 and 2019-20. In Bundelkhand agro-climatic region the activity of chickpea pod borer was observed from last week of December to last week of March while, its peak activity was noticed at $9^{\text {th }}, 10^{\text {th }}$ and $11^{\text {th }}$ SMW in chickpea agroecosystem. The infestation of chickpea pod borer (H. armigera) larvae was started from last week of December when the crop was in vegetative growth stage. Thereafter, its population increase gradually to reach maximum (1.72 larvae/mrl) at $10^{\text {th }}$ standard (first fortnight of March) after that its population declined gradually till the harvesting of the crop. The chickpea intercropped with safflower, mustard, linseed, barley and marigold had significant influence in reducing the larval population of chickpea pod borer ( $H$. armigera). The chickpea intercropped with marigold (0.71larva/mrl) and mustard $(0.75 \mathrm{larva} / \mathrm{mrl})$ was found the most effective in reducing the larval population of $H$. armigera. It was followed by chickpea + linseed, chickpea + barley and chickpea + safflower intercropping systems. The maximum population of chickpea pod borer was observed in chickpea sole crop (1.07larvae/mrl).

\section{Introduction}

Gram pod borer, Helicoverpa armigera (Hubner) is major pest of chickpea responsible for severe crop loss. Intercropping can affect micro climate of the agro ecosystem and ultimately produce an unfavorable environment for the pest. The impact of intercropping and other agro techniques on population build up of crop pest is influence by the changes in crop canopy and resulted change in microclimate (Singh et al., 2017). The practice of intercropping or mixed cropping of chickpea is common in the Indian Subcontinents and is a rule rather than an exception. Despite the predominance of low 
input traditional intercropping system in developing countries in the tropics such systems have received in adequate research attention. The trend in research on sole crop is still so strong that the agricultural scientists of most of the developing countries still pay little attention to research on intercropping system. Fortunately, there is a growing realization of the importance of catering to the need of the subsistence farmer and this demand more input in to the study of traditional system of crop production. The pest management through cropping system entomology is based on the sound principles of ecology and economics that cause considerable impact on population build up of insect pests and it also favored the activity of predators and parasitoids (Smith and Bosch, 1967). Hence, an effort was made to ascertain the influence of intercropping on larval population of $H$. armigera in chickpea.

\section{Materials and Methods}

The experiment was conducted was at Agricultural Research Farm of Brahmanand Post Graduate College, Rath, Hamirpur, U.P. during 2016-17 and 2019-20 cropping season. The field trail was laid out in Randomized Complete Block Design with three replications and five treatments including chickpea sole crop. The chickpea cultivar Radhey was intercropped with safflower $(2: 1)$, mustard (2:1), linseed (2:1), barley (2:1), and marigold (2:1). Monitoring of the experimental crop was done on regular basis to observe the infestation level of chickpea pod borer larvae. The observations were started as soon as the insect pests infestations were noticed in the field and collection of data were carried out at weekly intervals. The observation on population dynamics of $H$. armigera larvae was recorded randomly between 8 to $11 \mathrm{AM}$ and 3 to $5 \mathrm{PM}$. The absolute population of pod borer larvae was counted by thoroughly observing one meter row length of chickpea crop and replicated thrice in each experimental plot. The larval populations were subjected to square root transformation with the formula $\sqrt{X+0.5}$ as suggested by Heinrichs et al., (1981). The converted data were statistically calculated by following factorial randomized block design. The critical differences were calculated to ascertain the significance of difference among the various intercropping systems.

\section{Results and Discussion}

The incidence of chickpea pod borer in chickpea crop was started at vegetative stage in both the cropping season at $52^{\text {th }} \mathrm{SMW}$ is presented in table 1 and 2 . In the first year cropping season the maximum mean incidence of $H$. armigera was observed in chickpea sole crop (1.21 larvae/mrl) and the minimum mean incidence was recorded in chickpea + marigold intercropping system $(0.68$ larva/mrl) which was statistically at par with chickpea + mustard $(0.74$ larva/mrl $)$ intercrops. The intercropping system had significant influence in reduction of $H$. armigera population over to chickpea sole crop except chickpea + marigold and chickpea + mustard, chickpea + mustard and chickpea + linseed and chickpea + linseed and chickpea + barley were found to be at par with each other in their respective groups.

The appearance of $H$. armigera incidence among all the intercrops was observed at $52^{\text {th }}$ SMW with their minimum incidence $(0.28$ larva / mrl) thereafter, its population increased gradually to reached it peak (2.26 larvae/mrl) at $10^{\text {th }} \mathrm{SMW}$. The population density of $H$. armigera larvae more than 1 larva /mrl was recorded at flowering and pod formation stage $\left(7^{\text {th }}\right.$ SMW to $11^{\text {th }}$ SMW). The interaction effect of intercrops and periods of observation on the population of $H$. armigera larvae was fairly significant. In the second year of experimentation, the maximum number of pod borer larvae $(H$. armigera) was observed in 
chickpea sole crop (0.94 larva / mrl). While chickpea cultivar Radhey intercropped with marigold (0.74 larva / $\mathrm{mrl}$ ), had minimum infestation of gram pod borer it was followed by chickpea + mustard (0.76 larva/ mrl), chickpea + linseed (0.79 larva / $\mathrm{mrl})$ and chickpea + barley (0.82 larva / mrl) which were found to be at par with each other and varied significantly with chickpea sole crop (Table.2).

The maximum population of pod borer larva (1.43 larvae / mrl) was observed at 9 th standard meteorological week (SMW). The population of pod borer differed significantly at different periods of observation except the population at $2^{\text {nd }}, 3^{\text {rd }}, 4^{\text {th }}, 5^{\text {th }}, 6^{\text {th }}$ and $7^{\text {th }}$ SMW and $8^{\text {th }}, 9^{\text {th }}, 13^{\text {th }}, 14^{\text {th }}$ and $12^{\text {th }}$ SMW were found to be statistically at par with each other. The population at $9^{\text {th }}$ and $10^{\text {th }} \mathrm{SMW}$ was found the maximum during 2019-20 cropping season. The interaction effect of intercrops and period on the population of $H$. armigera larvae was significant.

The average of two years population data on pod borer observed in chickpea cultivar Radhey grown as intercrops during 2016-17 and 2019-20 is presented in table-3. The highest population of chickpea pod borer larvae was observed in chickpea sole crop (1.07 larvae / mrl).

The impact of intercropping on the population of chickpea pod borer was found to be significant except that recorded in the crop of chickpea intercropped with marigold and mustard, mustard and linseed and linseed and barley was found to be statistically at par with each other their respective groups.

The chickpea intercropped with marigold and mustard was found most effective in reducing the larval population of $H$. armigera. The minimum population was observed at $52^{\text {th }}$ SMW (0.27 larva / $\mathrm{mrl})$ at vegetative stage of crop. The larval population increased gradually to reached its peak (1.72 larvae / mrl) at $10^{\text {th }} \mathrm{SMW}$ followed by its population decreased to reached ( 0.48 larva / mrl) just prior to harvesting of the crop during second year of cropping season the $H$. armigera population above economic threshold level was observed at $9^{\text {th }} 10^{\text {th }}$ and $11^{\text {th }}$ SMW and varied significantly with other meteorological weeks of observation.

The chickpea intercropped with safflower, mustard, linseed, barley and marigold had significant influence on reduction of larval population as compared to chickpea sole crop during 2016-17 and 2019-20 cropping seasons. The minimum (0.28 and 0.26 larva / $\mathrm{mrl}$ ) population of gram pod borer was observed at $52^{\text {th }}$ SMW (standard meteorological week) during both the cropping season.

Thereafter, its population increased gradually to reached its peak (2.26 larvae/mrl and 1.43 larvae $/ \mathrm{mrl}$ ) at 10 days SMW and $9^{\text {th }}$ SMW, respectively during 2016-17 and 2019-20. After that its population decreased to reached its minimum (0.48and 0.91 larva/mrl) at $13^{\text {th }}$ SMW during both the cropping season.

The finding is corroborated with the earlier workers Chhatar et al., (2010) Waseem et al., (2017) amd Wasu et al., (2020) that reported the maximum activity of $H$. armigera was observed at $1^{\text {st }}$ fortnight of March in chickpea cropping system.

Among all the intercrops, chickpea intercropped with marigold was found most effective in reducing the larval population of $H$. armigera during both the cropping seasons and was found at par with chickpea + mustard intercrops. Their average population density was 0.71 and 0.75 larva / mrl. The average population density of $H$. armigera larvae (1.72 larvae $/ \mathrm{mrl}$ ) was observed at $10^{\text {th }} \mathrm{SMW}$. 
Table.1 Influence of intercropping systems on larval population of chickpea pod borer ( Helicoverpa armigera) during 2016-17 cropping season

\begin{tabular}{|c|c|c|c|c|c|c|c|c|c|c|c|c|c|c|c|}
\hline \multirow[t]{2}{*}{ Intercrops } & \multicolumn{14}{|c|}{ Population /metre row length } & \multirow[t]{2}{*}{ Average } \\
\hline & $\begin{array}{c}\text { SMW } \\
52\end{array}$ & $\begin{array}{c}\text { SMW } \\
1\end{array}$ & $\begin{array}{c}\text { SMW } \\
2\end{array}$ & $\begin{array}{c}\text { SMW } \\
\mathbf{3}\end{array}$ & $\begin{array}{c}\text { SMW } \\
4\end{array}$ & $\begin{array}{c}\text { SMW } \\
5\end{array}$ & $\begin{array}{c}\text { SMW } \\
6\end{array}$ & $\begin{array}{c}\text { SMW } \\
7\end{array}$ & $\begin{array}{c}\text { SMW } \\
8\end{array}$ & $\begin{array}{c}\text { SMW } \\
9\end{array}$ & $\begin{array}{c}\text { SMW } \\
10\end{array}$ & $\begin{array}{c}\text { SMW } \\
11\end{array}$ & $\begin{array}{c}\text { SMW } \\
12\end{array}$ & $\begin{array}{c}\text { SMW } \\
13\end{array}$ & \\
\hline $\begin{array}{c}\text { Chickpea+safflower } \\
\text { (T1) }\end{array}$ & $\begin{array}{c}0.45 \\
(0.94)\end{array}$ & $\begin{array}{c}0.33 \\
(0.88)\end{array}$ & $\begin{array}{c}0.33 \\
(0.86)\end{array}$ & $\begin{array}{c}0.33 \\
(0.88)\end{array}$ & $\begin{array}{c}0.56 \\
(0.99)\end{array}$ & $\begin{array}{c}0.89 \\
(1.12)\end{array}$ & $\begin{array}{c}1.44 \\
(1.36)\end{array}$ & $\begin{array}{c}1.33 \\
(1.31) \\
\end{array}$ & $\begin{array}{c}1.00 \\
(1.19) \\
\end{array}$ & $\begin{array}{c}1.22 \\
(1.29)\end{array}$ & $\begin{array}{c}2.67 \\
(1.69) \\
\end{array}$ & $\begin{array}{c}1.11 \\
(1.23)\end{array}$ & $\begin{array}{c}0.78 \\
(1.09)\end{array}$ & $\begin{array}{c}0.44 \\
(0.94) \\
\end{array}$ & $\begin{array}{c}0.92 \\
(1.13) \\
\end{array}$ \\
\hline $\begin{array}{c}\text { Chickpea+mustard } \\
\text { (T2) }\end{array}$ & $\begin{array}{c}0.11 \\
(0.77)\end{array}$ & $\begin{array}{c}0.44 \\
(0.94)\end{array}$ & $\begin{array}{c}0.22 \\
(0.82)\end{array}$ & $\begin{array}{c}0.44 \\
(0.94)\end{array}$ & $\begin{array}{c}0.44 \\
(0.92)\end{array}$ & $\begin{array}{c}0.67 \\
(1.05)\end{array}$ & $\begin{array}{c}0.33 \\
(0.88)\end{array}$ & $\begin{array}{c}1.44 \\
(1.37)\end{array}$ & $\begin{array}{c}0.89 \\
(1.15)\end{array}$ & $\begin{array}{c}1.11 \\
(1.23)\end{array}$ & $\begin{array}{c}2.11 \\
(1.56)\end{array}$ & $\begin{array}{c}1.11 \\
(1.24)\end{array}$ & $\begin{array}{c}0.78 \\
(1.07)\end{array}$ & $\begin{array}{c}0.22 \\
(0.82)\end{array}$ & $\begin{array}{c}0.74 \\
(1.05)\end{array}$ \\
\hline $\begin{array}{c}\text { Chickpea+linseed } \\
\text { (T3) }\end{array}$ & $\begin{array}{c}0.33 \\
(0.88)\end{array}$ & $\begin{array}{c}0.33 \\
(0.88)\end{array}$ & $\begin{array}{c}0.22 \\
(0.82)\end{array}$ & $\begin{array}{c}0.33 \\
(0.88)\end{array}$ & $\begin{array}{c}0.67 \\
(1.03)\end{array}$ & $\begin{array}{c}1.11 \\
(1.26)\end{array}$ & $\begin{array}{c}1.00 \\
(1.13)\end{array}$ & $\begin{array}{c}0.67 \\
(1.03)\end{array}$ & $\begin{array}{c}1.11 \\
(1.24)\end{array}$ & $\begin{array}{c}0.89 \\
(1.15)\end{array}$ & $\begin{array}{c}1.56 \\
(1.39)\end{array}$ & $\begin{array}{c}1.22 \\
(1.30)\end{array}$ & $\begin{array}{c}0.89 \\
(1.13)\end{array}$ & $\begin{array}{c}0.67 \\
(1.02)\end{array}$ & $\begin{array}{c}0.79 \\
(1.08)\end{array}$ \\
\hline $\begin{array}{c}\text { Chickpea+barley } \\
\text { (T4) }\end{array}$ & $\begin{array}{c}0.56 \\
(0.99) \\
\end{array}$ & $\begin{array}{c}0.33 \\
(0.88)\end{array}$ & $\begin{array}{c}0.78 \\
(1.09)\end{array}$ & $\begin{array}{c}0.56 \\
(0.99)\end{array}$ & $\begin{array}{c}0.78 \\
(1.09)\end{array}$ & $\begin{array}{c}0.44 \\
(0.94) \\
\end{array}$ & $\begin{array}{c}1.00 \\
(1.19)\end{array}$ & $\begin{array}{c}0.78 \\
(1.07) \\
\end{array}$ & $\begin{array}{c}0.89 \\
(1.13) \\
\end{array}$ & $\begin{array}{c}1.00 \\
(1.19)\end{array}$ & $\begin{array}{c}1.44 \\
(1.33) \\
\end{array}$ & $\begin{array}{c}1.33 \\
(1.34) \\
\end{array}$ & $\begin{array}{c}0.67 \\
(1.03)\end{array}$ & $\begin{array}{c}0.56 \\
(0.99) \\
\end{array}$ & $\begin{array}{c}0.79 \\
(1.09) \\
\end{array}$ \\
\hline $\begin{array}{c}\text { Chickpea+marigold } \\
\text { (T5) }\end{array}$ & $\begin{array}{c}0.11 \\
(0.77)\end{array}$ & $\begin{array}{c}0.33 \\
(0.88)\end{array}$ & $\begin{array}{c}0.22 \\
(0.82)\end{array}$ & $\begin{array}{c}0.45 \\
(0.94)\end{array}$ & $\begin{array}{c}0.22 \\
(0.82)\end{array}$ & $\begin{array}{c}0.78 \\
(1.05)\end{array}$ & $\begin{array}{c}0.78 \\
(1.07)\end{array}$ & $\begin{array}{c}0.78 \\
(1.07)\end{array}$ & $\begin{array}{c}0.89 \\
(1.15)\end{array}$ & $\begin{array}{c}0.56 \\
(0.99)\end{array}$ & $\begin{array}{c}2.22 \\
(1.59)\end{array}$ & $\begin{array}{c}1.00 \\
(1.20)\end{array}$ & $\begin{array}{c}0.78 \\
(1.11)\end{array}$ & $\begin{array}{c}0.33 \\
(0.88)\end{array}$ & $\begin{array}{c}0.68 \\
(1.02)\end{array}$ \\
\hline $\begin{array}{c}\text { Chickpea } \\
\text { Sole crop (T6) }\end{array}$ & $\begin{array}{c}0.11 \\
(0.77)\end{array}$ & $\begin{array}{c}0.22 \\
(0.82)\end{array}$ & $\begin{array}{c}0.78 \\
(1.06)\end{array}$ & $\begin{array}{c}0.78 \\
(1.11)\end{array}$ & $\begin{array}{c}0.44 \\
(0.92)\end{array}$ & $\begin{array}{c}1.11 \\
(1.22)\end{array}$ & $\begin{array}{c}0.78 \\
(1.11)\end{array}$ & $\begin{array}{c}1.33 \\
(1.32)\end{array}$ & $\begin{array}{c}1.67 \\
(1.45)\end{array}$ & $\begin{array}{c}2.33 \\
(1.66)\end{array}$ & $\begin{array}{c}3.56 \\
(1.99)\end{array}$ & $\begin{array}{c}1.67 \\
(1.45)\end{array}$ & $\begin{array}{c}1.44 \\
(1.38)\end{array}$ & $\begin{array}{c}0.67 \\
(0.99)\end{array}$ & $\begin{array}{c}1.21 \\
(1.23)\end{array}$ \\
\hline Average & $\begin{array}{c}0.28 \\
(0.85)\end{array}$ & $\begin{array}{c}0.33 \\
(0.88)\end{array}$ & $\begin{array}{c}0.43 \\
(0.91)\end{array}$ & $\begin{array}{c}0.48 \\
(0.96) \\
\end{array}$ & $\begin{array}{c}0.52 \\
(0.96)\end{array}$ & $\begin{array}{c}0.83 \\
(1.11)\end{array}$ & $\begin{array}{c}0.89 \\
(1.12)\end{array}$ & $\begin{array}{c}1.06 \\
(1.20)\end{array}$ & $\begin{array}{c}1.08 \\
(1.22)\end{array}$ & $\begin{array}{c}1.19 \\
(1.25)\end{array}$ & $\begin{array}{c}2.26 \\
(1.59)\end{array}$ & $\begin{array}{c}1.24 \\
(1.29)\end{array}$ & $\begin{array}{c}0.89 \\
(1.14)\end{array}$ & $\begin{array}{c}0.48 \\
(0.94) \\
\end{array}$ & $\begin{array}{c}0.85 \\
(1.10) \\
\end{array}$ \\
\hline \multicolumn{16}{|c|}{$\begin{array}{l}\text { Figures in the parenthesis are transformed as } \\
\text { Difference between intercrops } C D(P=0.05)=0.0 \\
\text { Difference between periods } C D(P=0.05)=0.04 \\
\text { Interaction between intercrops } x \text { periods } C D(P\end{array}$} \\
\hline
\end{tabular}


Table.2 Influence of intercropping systems on larval population of chickpea pod borer (Helicoverpa armigera) during 2019-20 cropping season

\begin{tabular}{|c|c|c|c|c|c|c|c|c|c|c|c|c|c|c|c|}
\hline \multirow[t]{2}{*}{ Intercrops } & \multicolumn{14}{|c|}{ Population /metre row length } & \multirow[t]{2}{*}{ Average } \\
\hline & $\begin{array}{c}\text { SMW } \\
52\end{array}$ & $\begin{array}{c}\text { SMW } \\
1\end{array}$ & $\begin{array}{c}\text { SMW } \\
2\end{array}$ & $\begin{array}{l}\text { SMW } \\
\mathbf{3}\end{array}$ & $\begin{array}{c}\text { SMW } \\
4\end{array}$ & $\begin{array}{l}\text { SMW } \\
5\end{array}$ & $\begin{array}{l}\text { SMW } \\
6\end{array}$ & $\begin{array}{c}\text { SMW } \\
7\end{array}$ & $\begin{array}{l}\text { SMW } \\
8\end{array}$ & $\begin{array}{l}\text { SMW } \\
9\end{array}$ & $\begin{array}{c}\text { SMW } \\
10\end{array}$ & $\begin{array}{c}\text { SMW } \\
11\end{array}$ & $\begin{array}{c}\text { SMW } \\
12\end{array}$ & $\begin{array}{c}\text { SMW } \\
13\end{array}$ & \\
\hline $\begin{array}{c}\text { Chickpea+safflower } \\
\text { (T1) }\end{array}$ & $\begin{array}{c}0.11 \\
(0.77)\end{array}$ & $\begin{array}{c}0.56 \\
(0.99)\end{array}$ & $\begin{array}{c}0.78 \\
(1.09)\end{array}$ & $\begin{array}{c}0.78 \\
(1.09)\end{array}$ & $\begin{array}{c}0.67 \\
(1.05)\end{array}$ & $\begin{array}{c}0.67 \\
(1.03)\end{array}$ & $\begin{array}{c}0.67 \\
(1.05)\end{array}$ & $\begin{array}{c}0.89 \\
(1.15)\end{array}$ & $\begin{array}{c}1.00 \\
(1.19)\end{array}$ & $\begin{array}{c}1.78 \\
(1.48)\end{array}$ & $\begin{array}{c}1.00 \\
(1.19)\end{array}$ & $\begin{array}{c}1.11 \\
(1.24)\end{array}$ & $\begin{array}{c}1.00 \\
(1.20)\end{array}$ & $\begin{array}{c}1.00 \\
(1.19)\end{array}$ & $\begin{array}{c}0.86 \\
(1.12)\end{array}$ \\
\hline $\begin{array}{c}\text { Chickpea+mustard } \\
\text { (T2) }\end{array}$ & $\begin{array}{c}0.11 \\
(0.77)\end{array}$ & $\begin{array}{c}0.44 \\
(0.94)\end{array}$ & $\begin{array}{c}0.33 \\
(0.88)\end{array}$ & $\begin{array}{c}0.67 \\
(1.03)\end{array}$ & $\begin{array}{c}0.44 \\
(0.94)\end{array}$ & $\begin{array}{c}0.67 \\
(1.03)\end{array}$ & $\begin{array}{c}0.55 \\
(0.99)\end{array}$ & $\begin{array}{c}0.78 \\
(1.09)\end{array}$ & $\begin{array}{c}0.78 \\
(1.07)\end{array}$ & $\begin{array}{c}1.44 \\
(1.36)\end{array}$ & $\begin{array}{c}1.22 \\
(1.27)\end{array}$ & $\begin{array}{c}1.11 \\
(1.24)\end{array}$ & $\begin{array}{c}0.89 \\
(1.13)\end{array}$ & $\begin{array}{c}1.22 \\
(1.30)\end{array}$ & $\begin{array}{c}0.76 \\
(1.07)\end{array}$ \\
\hline $\begin{array}{l}\text { Chickpea+linseed } \\
\text { (T3) }\end{array}$ & $\begin{array}{c}0.22 \\
(0.82)\end{array}$ & $\begin{array}{c}0.78 \\
(1.09)\end{array}$ & $\begin{array}{c}0.56 \\
(0.99)\end{array}$ & $\begin{array}{c}0.56 \\
(0.98)\end{array}$ & $\begin{array}{c}0.67 \\
(1.03)\end{array}$ & $\begin{array}{c}0.78 \\
(1.09)\end{array}$ & $\begin{array}{c}0.67 \\
(1.05)\end{array}$ & $\begin{array}{c}0.89 \\
(1.15)\end{array}$ & $\begin{array}{c}0.89 \\
(1.11)\end{array}$ & $\begin{array}{c}1.00 \\
(1.19)\end{array}$ & $\begin{array}{c}1.22 \\
(1.27)\end{array}$ & $\begin{array}{c}1.00 \\
(1.17)\end{array}$ & $\begin{array}{c}1.00 \\
(1.17)\end{array}$ & $\begin{array}{c}0.78 \\
(1.09)\end{array}$ & $\begin{array}{c}0.79 \\
(1.09)\end{array}$ \\
\hline $\begin{array}{c}\text { Chickpea+barley } \\
\text { (T4) }\end{array}$ & $\begin{array}{c}0.44 \\
(0.94)\end{array}$ & $\begin{array}{c}0.55 \\
(0.98)\end{array}$ & $\begin{array}{c}0.67 \\
(1.03)\end{array}$ & $\begin{array}{c}0.89 \\
(1.15)\end{array}$ & $\begin{array}{c}0.89 \\
(1.15)\end{array}$ & $\begin{array}{c}0.45 \\
(0.94)\end{array}$ & $\begin{array}{c}1.00 \\
(1.19)\end{array}$ & $\begin{array}{c}0.67 \\
(1.02)\end{array}$ & $\begin{array}{c}0.56 \\
(0.98)\end{array}$ & $\begin{array}{l}1.55 \\
(1.40)\end{array}$ & $\begin{array}{c}1.45 \\
(1.36)\end{array}$ & $\begin{array}{c}0.44 \\
(0.94)\end{array}$ & $\begin{array}{c}1.00 \\
(1.20)\end{array}$ & $\begin{array}{c}0.89 \\
(1.13)\end{array}$ & $\begin{array}{c}0.82 \\
(1.10)\end{array}$ \\
\hline $\begin{array}{c}\text { Chickpea+marigold } \\
\text { (T5) }\end{array}$ & $\begin{array}{c}0.33 \\
(0.88)\end{array}$ & $\begin{array}{c}0.67 \\
(1.03)\end{array}$ & $\begin{array}{c}1.11 \\
(1.24)\end{array}$ & $\begin{array}{c}0.33 \\
(0.88)\end{array}$ & $\begin{array}{c}0.44 \\
(0.94)\end{array}$ & $\begin{array}{c}0.89 \\
(1.15)\end{array}$ & $\begin{array}{c}0.33 \\
(0.86)\end{array}$ & $\begin{array}{c}1.00 \\
(1.19)\end{array}$ & $\begin{array}{c}0.89 \\
(1.13)\end{array}$ & $\begin{array}{c}1.11 \\
(1.22)\end{array}$ & $\begin{array}{c}1.00 \\
(1.19)\end{array}$ & $\begin{array}{c}0.89 \\
(1.15)\end{array}$ & $\begin{array}{c}0.89 \\
(1.13)\end{array}$ & $\begin{array}{c}0.44 \\
(0.94)\end{array}$ & $\begin{array}{c}0.74 \\
(1.07)\end{array}$ \\
\hline $\begin{array}{c}\text { Chickpea Sole crop } \\
\text { (T6) }\end{array}$ & $\begin{array}{c}0.33 \\
(0.88)\end{array}$ & $\begin{array}{c}0.89 \\
(1.15)\end{array}$ & $\begin{array}{c}0.67 \\
(1.03)\end{array}$ & $\begin{array}{c}0.98 \\
(1.15)\end{array}$ & $\begin{array}{c}1.00 \\
(1.17)\end{array}$ & $\begin{array}{c}0.78 \\
(1.09)\end{array}$ & $\begin{array}{c}1.11 \\
(1.24)\end{array}$ & $\begin{array}{c}0.67 \\
(1.03)\end{array}$ & $\begin{array}{c}1.00 \\
(1.19)\end{array}$ & $\begin{array}{c}1.67 \\
(1.45)\end{array}$ & $\begin{array}{c}1.11 \\
(1.23)\end{array}$ & $\begin{array}{c}1.00 \\
(1.19)\end{array}$ & $\begin{array}{c}0.78 \\
(1.09)\end{array}$ & $\begin{array}{c}1.11 \\
(1.23)\end{array}$ & $\begin{array}{c}0.94 \\
(1.21)\end{array}$ \\
\hline Average & $\begin{array}{c}0.26 \\
(0.84)\end{array}$ & $\begin{array}{c}0.65 \\
(1.03)\end{array}$ & $\begin{array}{c}0.69 \\
(1.04)\end{array}$ & $\begin{array}{c}0.69 \\
(1.05) \\
\end{array}$ & $\begin{array}{c}0.69 \\
(1.05)\end{array}$ & $\begin{array}{c}0.71 \\
(1.06)\end{array}$ & $\begin{array}{c}0.72 \\
(1.06)\end{array}$ & $\begin{array}{c}0.82 \\
(1.11)\end{array}$ & $\begin{array}{c}0.85 \\
(1.11)\end{array}$ & $\begin{array}{c}1.43 \\
(1.35) \\
\end{array}$ & $\begin{array}{c}1.17 \\
(1.25)\end{array}$ & $\begin{array}{c}0.93 \\
(1.16)\end{array}$ & $\begin{array}{c}0.93 \\
(1.15) \\
\end{array}$ & $\begin{array}{c}0.91 \\
(1.15)\end{array}$ & $\begin{array}{c}0.82 \\
(1.11)\end{array}$ \\
\hline \multicolumn{16}{|c|}{$\begin{array}{l}\text { Figures in the parenthesis are transformed as } \sqrt{X+0.5} \\
\text { Difference between intercrops } C D(P=0.05)=0.03 \\
\text { Difference between periods } C D(P=0.05)=0.05 \\
\text { Interaction between intercrops } x \text { periods } C D(P=0.05)=0.12\end{array}$} \\
\hline
\end{tabular}


Table.3 Influence of intercropping systems on larval population chickpea pod borer (Helicoverpa armigera) during 2016-17 and 2019-20 cropping season (Average)

\begin{tabular}{|c|c|c|c|c|c|c|c|c|c|c|c|c|c|c|c|}
\hline \multirow[b]{2}{*}{ Intercrops } & \multicolumn{14}{|c|}{ Population /metre row length } & \multirow[t]{2}{*}{ Average } \\
\hline & $\begin{array}{c}\text { SMW } \\
52\end{array}$ & $\begin{array}{c}\text { SMW } \\
1\end{array}$ & $\begin{array}{c}\text { SMW } \\
2\end{array}$ & $\begin{array}{c}\text { SMW } \\
\mathbf{3}\end{array}$ & $\begin{array}{c}\text { SMW } \\
4\end{array}$ & $\begin{array}{c}\text { SMW } \\
5\end{array}$ & $\begin{array}{c}\text { SMW } \\
6\end{array}$ & $\begin{array}{c}\text { SMW } \\
7\end{array}$ & $\begin{array}{c}\text { SMW } \\
8\end{array}$ & $\begin{array}{c}\text { SMW } \\
9\end{array}$ & $\begin{array}{c}\text { SMW } \\
10\end{array}$ & $\begin{array}{c}\text { SMW } \\
11\end{array}$ & $\begin{array}{c}\text { SMW } \\
12\end{array}$ & $\begin{array}{c}\text { SMW } \\
13\end{array}$ & \\
\hline $\begin{array}{c}\text { Chickpea } \\
\text { +safflower } \\
\text { (T1) }\end{array}$ & $\begin{array}{c}0.28 \\
(0.86)\end{array}$ & $\begin{array}{l}0.45 \\
(0.94)\end{array}$ & $\begin{array}{r}0.56 \\
(0.98)\end{array}$ & $\begin{array}{r}0.56 \\
(0.99)\end{array}$ & $\begin{array}{c}0.62 \\
(1.02)\end{array}$ & $\begin{array}{r}0.78 \\
(1.08)\end{array}$ & $\begin{array}{r}1.06 \\
(1.21)\end{array}$ & $\begin{array}{c}1.11 \\
(1.23)\end{array}$ & $\begin{array}{r}1.00 \\
(1.19)\end{array}$ & $\begin{array}{c}1.5 \\
1.39\end{array}$ & $\begin{array}{c}1.84 \\
(1.44)\end{array}$ & $\begin{array}{c}1.11 \\
(1.24)\end{array}$ & $\begin{array}{r}0.89 \\
(1.15)\end{array}$ & $\begin{array}{c}0.72 \\
(1.07)\end{array}$ & $\begin{array}{c}0.89 \\
(1.13)\end{array}$ \\
\hline $\begin{array}{c}\text { Chickpea } \\
+ \text { mustard } \\
\text { (T2) }\end{array}$ & $\begin{array}{l}0.11 \\
(0.77)\end{array}$ & $\begin{array}{l}0.44 \\
(0.94)\end{array}$ & $\begin{array}{l}0.28 \\
(0.85)\end{array}$ & $\begin{array}{l}0.56 \\
(0.99)\end{array}$ & $\begin{array}{l}0.44 \\
(0.93)\end{array}$ & $\begin{array}{l}0.67 \\
(1.04)\end{array}$ & $\begin{array}{l}0.44 \\
(0.94)\end{array}$ & $\begin{array}{l}1.11 \\
(1.23)\end{array}$ & $\begin{array}{l}0.84 \\
(1.11)\end{array}$ & $\begin{array}{l}1.28 \\
(1.30)\end{array}$ & $\begin{array}{l}1.67 \\
(1.42)\end{array}$ & $\begin{array}{l}1.11 \\
(1.24)\end{array}$ & $\begin{array}{l}0.84 \\
(1.10)\end{array}$ & $\begin{array}{l}0.72 \\
(1.06)\end{array}$ & $\begin{array}{l}0.75 \\
(1.07)\end{array}$ \\
\hline $\begin{array}{c}\text { Chickpea } \\
+ \text { linseed } \\
\text { (T3) }\end{array}$ & $\begin{array}{l}0.28 \\
(0.85)\end{array}$ & $\begin{array}{l}0.56 \\
(0.99)\end{array}$ & $\begin{array}{l}0.39 \\
(0.91)\end{array}$ & $\begin{array}{l}0.45 \\
(0.93)\end{array}$ & $\begin{array}{l}0.67 \\
(1.03)\end{array}$ & $\begin{array}{l}0.95 \\
(1.18)\end{array}$ & $\begin{array}{l}0.84 \\
(1.09)\end{array}$ & $\begin{array}{l}0.78 \\
(1.09)\end{array}$ & $\begin{array}{l}1.00 \\
(1.18)\end{array}$ & $\begin{array}{l}0.95 \\
(1.17)\end{array}$ & $\begin{array}{l}1.39 \\
(1.33)\end{array}$ & $\begin{array}{l}1.11 \\
(1.24)\end{array}$ & $\begin{array}{l}0.95 \\
(1.15)\end{array}$ & $\begin{array}{l}0.73 \\
(1.06)\end{array}$ & $\begin{array}{l}0.79 \\
(1.09)\end{array}$ \\
\hline $\begin{array}{c}\text { Chickpea } \\
\text { + barley } \\
\text { (T4) }\end{array}$ & $\begin{array}{l}0.50 \\
(0.97)\end{array}$ & $\begin{array}{l}0.44 \\
(0.93)\end{array}$ & $\begin{array}{l}0.73 \\
(1.06)\end{array}$ & $\begin{array}{l}0.73 \\
(1.07)\end{array}$ & $\begin{array}{l}0.84 \\
(1.12)\end{array}$ & $\begin{array}{l}0.45 \\
(0.94)\end{array}$ & $\begin{array}{l}1.00 \\
(1.19)\end{array}$ & $\begin{array}{l}0.73 \\
(1.05)\end{array}$ & $\begin{array}{l}0.73 \\
(1.06)\end{array}$ & $\begin{array}{l}1.28 \\
(1.30)\end{array}$ & $\begin{array}{l}1.45 \\
(1.35)\end{array}$ & $\begin{array}{l}0.89 \\
(1.14)\end{array}$ & $\begin{array}{l}0.84 \\
(1.12)\end{array}$ & $\begin{array}{l}0.73 \\
(1.06)\end{array}$ & $\begin{array}{l}0.81 \\
(1.10)\end{array}$ \\
\hline $\begin{array}{c}\text { Chickpea } \\
+ \\
\text { marigold } \\
\text { (T5) }\end{array}$ & $\begin{array}{l}0.22 \\
(0.83)\end{array}$ & $\begin{array}{l}0.50 \\
(0.96)\end{array}$ & $\begin{array}{l}0.67 \\
(1.03)\end{array}$ & $\begin{array}{l}0.39 \\
(0.91)\end{array}$ & $\begin{array}{l}0.33 \\
(0.88)\end{array}$ & $\begin{array}{l}0.84 \\
(1.10)\end{array}$ & $\begin{array}{l}0.56 \\
(0.97)\end{array}$ & $\begin{array}{l}0.89 \\
(1.13)\end{array}$ & $\begin{array}{l}0.89 \\
(1.14)\end{array}$ & $\begin{array}{l}0.84 \\
(1.11)\end{array}$ & $\begin{array}{l}1.61 \\
(1.39)\end{array}$ & $\begin{array}{l}0.95 \\
(1.18)\end{array}$ & $\begin{array}{l}0.84 \\
(1.12)\end{array}$ & $\begin{array}{l}0.39 \\
(0.91)\end{array}$ & $\begin{array}{l}0.71 \\
(1.05)\end{array}$ \\
\hline $\begin{array}{c}\text { Chickpea } \\
\text { Sole crop } \\
\text { (T6) }\end{array}$ & $\begin{array}{l}0.22 \\
(0.83)\end{array}$ & $\begin{array}{l}0.56 \\
(0.99)\end{array}$ & $\begin{array}{l}0.73 \\
(1.05)\end{array}$ & $\begin{array}{l}0.88 \\
(1.13)\end{array}$ & $\begin{array}{l}0.72 \\
(1.05)\end{array}$ & $\begin{array}{l}0.95 \\
(1.16)\end{array}$ & $\begin{array}{l}0.95 \\
(1.18)\end{array}$ & $\begin{array}{l}1.00 \\
(1.18)\end{array}$ & $\begin{array}{l}1.34 \\
1.32\end{array}$ & $\begin{array}{l}2.00 \\
(1.56)\end{array}$ & $\begin{array}{l}2.34 \\
(1.61)\end{array}$ & $\begin{array}{l}1.34 \\
(1.32)\end{array}$ & $\begin{array}{l}1.11 \\
(1.24)\end{array}$ & $\begin{array}{l}0.89 \\
(1.11)\end{array}$ & $\begin{array}{l}1.07 \\
(1.23)\end{array}$ \\
\hline Average & $\begin{array}{l}0.27 \\
(0.85)\end{array}$ & $\begin{array}{l}0.49 \\
(0.96)\end{array}$ & $\begin{array}{l}0.56 \\
(0.98)\end{array}$ & $\begin{array}{l}0.59 \\
(1.01)\end{array}$ & $\begin{array}{l}0.61 \\
(1.01)\end{array}$ & $\begin{array}{l}0.77 \\
(1.09)\end{array}$ & $\begin{array}{l}0.81 \\
(1.09)\end{array}$ & $\begin{array}{l}0.94 \\
(1.16)\end{array}$ & $\begin{array}{l}0.97 \\
(1.17)\end{array}$ & $\begin{array}{l}1.31 \\
(1.30)\end{array}$ & $\begin{array}{l}1.72 \\
(1.42)\end{array}$ & $\begin{array}{l}1.09 \\
(1.23)\end{array}$ & $\begin{array}{l}0.91 \\
(1.15)\end{array}$ & $\begin{array}{l}0.70 \\
(1.05)\end{array}$ & $\begin{array}{l}0.84 \\
(1.11)\end{array}$ \\
\hline \multicolumn{16}{|c|}{ Figures in the parenthesis are transformed as $\sqrt{X+0.5}$} \\
\hline
\end{tabular}


The chickpea inter cropped with mustard and linseed and linseed and barley were found statistically at par with each other in their respective groups but varied significantly from chickpea sole crop which had maximum infestation (1.07 larvae / mrl) of chickpea pod borer larvae. The present findings are in accordance with the earlier report made by Nath and Chakravorty (2005), Singh (2014), Waseem et al., (2017) and Patil (2018) that reported the chickpea intercropped with marigold, mustard, and barley. Linseed harboured the minimum population of chickpea pod borer larvae and varied significantly with chickpea sole crop.

\section{Acknowledgement}

The research paper is part of Ph.D thesis of Hari Prakash Namdev. The author is greatful to Principal B.N.P.G.college, Rath, Hamirpur (U.P.) for providing of Laboratory, Library and research field facilities. The author also oblige to Ph.D. research cell of Bundelkhand University, Jhansi for their valuable support and guidance.

\section{References}

Chatar,V. P., Raghvani, K. L., Joshi, M. D., Ghadge, S. M., Deshmukh, S. G. and Dalave S. K.(2010) Population dynamics of pod borer, Helicoverpa armigera (Hubner) infesting chickpea. International Journal of Plant Protection, 3 (1): 65-67

Heinrichs, E. A., Chelliah, S., Valencia, S. C., Arceo, M. B., Fabellar, L. T., Aquino, G. B. and Pickin, S.(1981) Statistical analysis of insect population and plant damage. Manual for testing insecticides on rice. I.R.R.I., Manila, Philippines, pp. 73-80.

Nath P. and Chakravorty.S.(2005) Effect of intercropping on the infestation of chickpea pod borer [Helicoverpa armigera (Hubner)].Journal of Plant Protection and Environment, 2 (1): 8691

Patil, A. A., Thakur, S., Navale, J. S., Narode, M. K. and Kolhe, P. S.(2018) Influence of intercropping on incidence of gram Pod borer (Helicoverpa armigera) in chickpea (Cicer arietinum L.) Journal of Entomology and Zoolsgy Studies. 6(5): 12-15

Singh, S. K.(2014) Usefulness of intercrops in management of African Bollworm, Helicoverpa armigera Hubner in chickpea, Cicer arietinum L. in Ethiopia. Journal of Food Legumes 27(3): 226229

Singh, Kuldeep., Raju, S. V. S. and Singh, N. N.(2015) Impact of different intercropping in the management of pod borer, Helicoverpa armigera Hüb. in chickpea. Journal of Entomological Research. 39 (3):219-222

Singh, R. S., Nath, P. and Kumar A. (2017) Effect of intercropping on population dynamic of pod borer complex of pigeon pea. New agriculturist.28 (1)235-238

Smith, R. F. and Bosch, Van den.(1967) Integrated Control. In:Kilgore,W.W.and Doutt,R.L.(ed.) Pest Control, Biological, Physical and Selected Methods. Academic Press, New York

Waseem, M. A., Simon, S. and Thakur, S. (2017) Effect of Intercropping on the Incidence of Gram Pod Borer, Helicoverpa armigera in Chickpea. Int.J.Curr.Microbiol.App.Sci. 6(10): 2619-2624

Wasu, R. S., Tathode, V. R. and Girhepunje, D. Y. (2020) Influence of plant density of marigold on larval population of gram pod borer $H$. armigera in chickpea (Cicer arientinum L.) International Journal of Chemical Studies, 8(6): 15231526. 


\section{How to cite this article:}

Hari Prakash Namdev, Ram Subhag Singh and Bharat Lal Kushwaha. 2021. Influence of Intercropping on Larval Population of Chickpea Pod Borer, Helicoverpa armigera (Hub.) in Chickpea (Cicer arietinum L.). Int.J.Curr.Microbiol.App.Sci. 10(09): 319-326. doi: https://doi.org/10.20546/ijcmas.2021.1009.037 